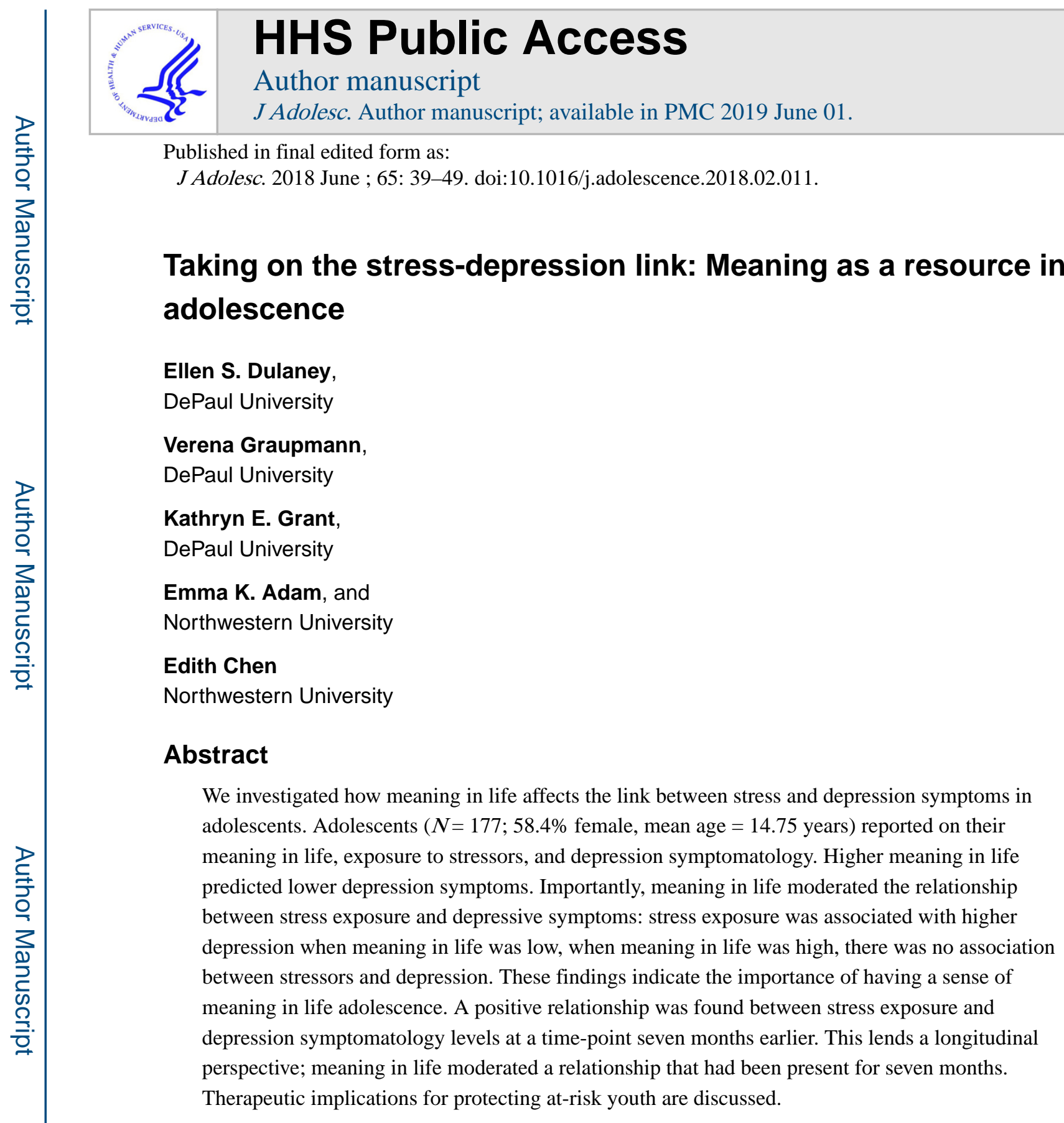

\title{
Keywords
}

life meaning; adolescence; stress; depression; coping

Taking on the stress-depression link: Meaning as a resource in adolescence Adolescence is a vulnerable period of development, during which youths are prone to experiencing stress and depression (Thapar, Collishaw, Pine, \& Thapar, 2012). Stress, a key factor in the etiology of depression (Hammen, 2005), is almost unavoidable for adolescents who by nature of their

Publisher's Disclaimer: This is a PDF file of an unedited manuscript that has been accepted for publication. As a service to our customers we are providing this early version of the manuscript. The manuscript will undergo copyediting, typesetting, and review of the resulting proof before it is published in its final citable form. Please note that during the production process errors may be discovered which could affect the content, and all legal disclaimers that apply to the journal pertain. 
developmental status face constant challenges of social role definition and increased responsibility in addition to other potential environmental strains (Steinberg \& Morris, 2001). Having meaning in life is associated with well-being in adolescents (Halama \& Dedová, 2007; Ho, Cheung, \& Cheung, 2010; Kiang \& Fuligni, 2010), suggesting that meaning in life may mitigate the negative effects of stressors on affective well-being. With the goal of integrating meaning in life as a resource into the stress-depression connection, here we examine directly how meaning in life affects the relationship between stress and depression in youths, specifically looking at relationships between meaning in life, stress exposure, and depressive symptomatology in adolescents.

\section{Stress and Depression}

Major depressive disorder consists of lowered mood and decreases in energy and emotional reactivity (American Psychiatric Association, 2013). It is a complex disorder, with a myriad of factors motivating its onset including genetic predisposition (Sullivan, Neale, \& Kendler, 2000), neurochemical regulation (Werner \& Coveñas, 2013), psychological schemas (Beck \& Haigh, 2014), and social and environmental circumstances (Monroe \& Hadjiyannakis, 2002). Among the environmental causes, chronic or extreme acute stress exposure is among the most heavily emphasized and researched. Numerous studies find major stressful events to precede the onset of depressive episodes (reviewed in Mazure, 1998), and have established a link between stress exposure and depression symptomatology (reviewed in Hammen, 2005; Monroe \& Hadjiyannakis, 2002; Paykel, 2003).

Models of the stress-depression relationship highlight the importance of how stressors are perceived cognitively. Under the generic cognitive model, a person's interpretations of stressors can create negative self-referential beliefs, schemas that bias information processing once activated (Beck \& Haigh, 2014). Accordingly, some cognitive appraisals of stressors are particularly likely to facilitate the development of depression (Beck \& Haigh, 2014; Disner, Beevers, Haigh, \& Beck, 2011). Meaning in life, as a culmination of cognitive and affective assessments of one's life, may weaken the role of cognitive appraisals in fostering depression.

\section{Meaning as a Buffer in the Stress-Depression Link}

Meaning in life refers to how coherent, purposeful, and significant a person feels their life to be. Synthesizing a vast literature, meaning in life can be defined as a person's ability to comprehend their life circumstances, their possession of motivating and life-organizing goals, and their feeling that their life is of importance (Martela \& Steger, 2016). Daily events are interpreted in their larger significance towards meaning in life, i.e. stressors can be evaluated for relevance to a person's overall understanding of the meaning of their life. Models of depression emphasize the appraisal of stressors in context of meaning in life to determine whether these stressors will lead to psychological harm (Beck \& Haigh, 2014). Negative views of the self, the world, and the future, known as the negative cognitive triad, have been associated with vulnerability for depression in adults (Beck \& Perkins, 2001) and in adolescents (Braet, Wante, Van Beveren, \& Theuwis, 2015), indicating the importance of a person's broad beliefs about their life and circumstances in mental health. 
To this end, having personal meaning in life structures may provide a context for understanding and integrating stressful situations. It is each person's ability to decide what makes their life meaningful. Thus, meaning in life represents a malleable cognitiveemotional framework, directly accessible to subjective evaluation based on one's own needs and values (Steger, 2009; Yalom, 1980). The pragmatic malleability of the meaning in life framework makes it a particularly promising route through which depression-facilitating cognitions may be resisted or reframed.

\section{Meaning in Life and Well-being}

Supporting the idea that meaning in life can be beneficial for psychological health, having meaning in life is widely regarded as critical for well-being (Steger et al., 2006). Meaning in life has been positively associated with self-satisfaction (Reker \& Cousins, 1979), life satisfaction (Park, Park, \& Peterson, 2010; Reker, 1977), acceptance of death (Durlak, 1972), happiness (Park et al., 2010), and subjective well-being (Zika \& Chamberlain, 1987; Zika \& Chamberlain, 1992). While investigations regarding the importance of existential questions in adolescent samples have been sparse (Brassai, Piko, \& Steger, 2012), recent studies indicate that meaning in life can promote adolescent well-being in the United States (Kiang \& Fuligni, 2010) and other countries (Halama \& Dedová, 2007; Ho et al., 2010; Hong, 2008; Tavernier \& Willoughby, 2012).

In addition to being conducive to well-being, meaning in life appears to help people avoid depressive symptomatology. People who report higher life meaning endorse lower depressive symptomatology (Kleftaras \& Psarra, 2012; Mascaro \& Rosen, 2008; Mascaro \& Rosen, 2005; Park et al., 2010). This effect has been shown in a variety of populations including HIV patients (Lyon \& Younger, 2001), cancer survivors (Simonelli, Fowler, Maxwell, \& Andersen, 2008), and clinically depressed persons (Thakur \& Basu, 2010). Interventions for increasing meaning in life have repeatedly shown to lower depression (Bohlmeijer, Smit, \& Cuijpers, 2003; Cho, Bernstein, Roh, \& Chen, 2013; Hsieh \& Wang, 2003).

In addition to the associations of meaning in life with improved well-being and resistance to depression, it has also been directly associated with lessened stressor impact. Meaning in life predicts decreases in symptoms of posttraumatic stress disorder in adults (PTSD; Owens, Steger, Whitesell, \& Herrera, 2009), and discovering meaning in life from trauma and combat events predicts decreased PTSD symptomatology (Currier, Holland, Chisty, \& Allen, 2011; Updegraff, Silver, \& Holman, 2008). Further, interventions to increase meaning in life have been demonstrated to improve PTSD symptoms and aid coping. Meaning interventions, focused on resolving religious discomfort and encouraging religious meaning-making, have been successfully applied to treat combat-related PTSD in survivors of military trauma (Harris et al., 2011).

Additional support for the connection between meaning in life and stress resistance exists in the coping literature. Much attention has been given to the benefits of posttraumatic growth (PTG), defined as obtaining a greater understanding of one's life, values, spirituality, and personal strength after experiencing a trauma (Tedeschi \& Calhoun, 2004). Meaning-making 
coping, coping with stressors by exploring how the stressor contains and can lead to meaning in life, is a central mechanism in the process of PTG in trauma survivors (Larner \& Blow, 2011). Adaptively creating life meaning out of an experience with cancer has been related to better psychological adjustment in cancer survivors (Park, Edmondson, Fenster, \& Blank, 2008). Meaning-making coping has been shown to mediate the relationship between religion and college students' adjustment to loss (Park, 2005), indicating that religion influences loss adjustment by providing a source of meaning in life. There is also evidence that beneficial emotional support from social ties functions partially by reinforcing one's sense of meaning in life (Krause, 2004). This demonstrates that meaning in life may underlie some factors in the coping process. The association of meaning in life with well-being and resilience implies a role of meaning in life for resisting stressor impact, a role that may be especially suited to support coping with the challenges of transition in adolescence.

\section{Meaning and Adolescents' Stressor Coping}

In light of this multitude of empirical findings highlighting the importance of meaning in life for well-being, meaning in life emerges as a strong candidate for psychological coping with stress. Thus far, to our knowledge, the stress-moderating role of meaning in life for coping specifically during adolescence remains unexplored in the literature. There are theoretical reasons to expect that meaning in life would have such a stress-moderating role for adolescents. As a malleable, self-evaluated, cognitive-emotional framework (Steger, 2009), meaning in life may be an avenue through which the cognitive importance of stressors can be reframed. This would not be an insignificant buffer against stressors, considering that a large part of a stressor's threat to well-being is made up of its relevance for cognitive selfschemas (Beck \& Haigh, 2014; Disner et al., 2011).

Further, meaning in life seems an especially useful tool for those who experience stressors that they have limited direct control over, as is the case with adolescents. Orienting to stressors adaptively may be important in resisting depression, particularly for those vulnerable or with low direct control over their circumstances. In adolescence, stress is a regular component of life as adolescents face physiological and emotional changes in addition to dramatic role restructuring at the familial, peer, and community levels (Eccles, Templeton, Barber, \& Stone, 2003; Steinberg \& Morris, 2001). Since adolescents cannot necessarily change the transitional events they experience, coping strategies that - instead of targeting these stressors directly - target related perceptions and cognitions may be particularly useful in cushioning stressor impact. In other words, meaning in life may be a useful shield against the impact of stressors that adolescents would otherwise have difficulty avoiding.

If adolescents can rely on personal meaning in life structures for stability in times of stress, e.g. confronting the challenges of this developmental period, their vulnerability for depressive symptoms may be reduced. Adolescents can conceivably employ meaning in life as an anchor in two ways. Adolescents may broadly benefit from the certainty that having a strong sense of their life's meaning provides. In this view, when confronted with a stressor meaning in life may provide the adolescent with a foundational context of stable goals, narratives, and feelings of personal importance that renders the individual stressor less 
significant and threatening. Alternately, adolescents may cope with a stressor by reflecting on its specific benefits for their life's meaning. Known as shifting in the literature, it is thought that people can engage in shifting to reframe a stressor in terms of its positive implications for the future (Chen, McLean, \& Miller, 2015). Specifically, shifting is the ability to reframe the significance of a stressor as less threatening in the big picture of an individual's goals and life meaning narratives.

In support of a role for meaning in life in adolescent stress resilience, meaning in life has been related to decreased inflammatory immune cell response to chronic stress in lowsocioeconomic status adolescents and their parents, a response associated with chronic disease (Chen et al., 2015). Notably, the anti-inflammatory function in response to chronic stress has been seen in participants high in both meaning in life and a tendency for shifting (Chen et al., 2015). The physiological interaction of meaning in life and shifting engagement suggests that when adolescents possess meaning in life, a connection to big-picture goals and systems, they may be better able to cope with stressors in the moment. This cements the need to explore levels of meaning in life as well as shifting tendencies as potential avenues for adolescent stress resistance.

\section{Current Study and Hypotheses}

Can meaning in life bolster adolescents against stress and depression by weakening the stress-depression link? Meaning in life may benefit youths by providing overall stability as well as cognitive belief structures that can be used for adaptively reframing specific stressors. We investigated these questions as part of a large research effort to assess adolescents' well-being, stressor reactivity, psychopathology, and resilience. The present study examines data from two data collection waves from this endeavor. We examined the relationship between stress and depression at the two time points, as well as the progression of stress exposure and depression over the two time points. Importantly, the questionnaires administered at the second wave contained items targeting meaning in life, allowing us to specifically examine the role of meaning in life and shifting in the stress-depression relationship. Thus, in addition to gaining a longitudinal perspective on the stress-depression relationship, we obtained cross-sectional insight into the role of meaning in life role in the stress-depression link at the second time point. The current study sought to test the hypothesis that meaning in life can buffer the impact of stress on depression as an outcome in adolescents. Furthermore, we explored whether shifting may buffer against stress and depression to better compare these two avenues through which adolescents may resist stressors.

\section{Method}

\section{Participants and Design}

Survey responses from 201 participants were used to examine our relationships of interest. These data were collected as part of a large research endeavor at a Midwestern university at two time points, seven months apart. Students were randomly assigned to an order of participation in various measures and tasks assessing life circumstances, mental and emotional well-being, and physiological reactivity to stressful situations. 391 adolescents 
(ages 11 to 19) were recruited from three diverse urban schools located in a major city in the Midwest (two schools with grades K- $8^{\text {th }}$; one high-school). The two data collections took place for an entire day each on six consecutive Saturdays, for the first wave and, seven months later, on five consecutive Saturdays for the second wave. Due to these limited windows for data collection many participants were not available to devote an entire second day to attend the second wave of data collection, resulting in substantial participation attrition. 201 participants were able to provide data at the second time point, and 177 participants provided data at both time points.

Out of participants' total responses, data from three survey measures represented our target stress exposure, depression symptoms, meaning in life, and shifting variables. Participants completed stress and depression measures at both time points, and completed the items representing meaning in life and shifting at the second data collection time point. The items targeting meaning in life and shifting were added to the large research survey at the second time point to more fully capture the stress coping process in adolescence and complement the battery of psychopathology, life experience, cognitions about stress, and physiological measures already included in the study seven months prior.

For our research goal of understanding the role of meaning in life for the stress coping process during adolescence, the Persist-subscale $\left(a_{\text {adolescents }}=.73\right)$ of the Shift-and-Persist Questionnaire (SAPQ; Chen et al., 2015) asking respondents to report on their sense of purpose in life seemed particularly well-suited. The SAPQ was designed specifically to evaluate responses to stress by reframing stressors with the Persist-subscale referring to personal meaning in life structures. Importantly the SAPQ has been validated for use in adolescent populations to evaluate physiological stressor response outcomes (Chen et al., 2015): Convergent validity $(r=0.81, p<.001)$ with the Purpose in Life Scale (Robbins \& Francis, 2000), which relates to the meaning-making process opposing meaninglessness, suggests a clear connection to the psychological concept of meaning. We further deemed the word "purpose" to be an appropriate way to capture adolescents' meaning in life, as purpose is a familiar and tangible term for this concept for adolescent understanding. Validating this rationale, the word "purpose" is also used in half of the items on the Meaning in Life Questionnaire (Steger et al., 2006), an instrument widely used to measure meaning in life. We thus judged the SAPQ to be an appropriate measure by which to begin probing the link between meaning in life and coping with stress, as it is in this specific use that this measure has been developed (Chen et al., 2015).

Our analyses below examine stress and depression correlation patterns at both time points using data only from participants who provided data at both times $(\mathrm{N}=177)$. Main analyses examined the impact of meaning in life and shifting in the stress-depression relationship using these longitudinally-matched individuals. These participants were $58.4 \%$ female (age $M=14.90$ years, range $=11-19$ years); $29.8 \%$ Black or African-American, $18.5 \%$ Hispanic or Latino/a, 11.2\% Asian or Asian-American, 10.1\% White or Caucasian, 0.6\% American Indian or Alaskan Native, 25.8\% Bi-Racial/Multi-Racial, 2.8\% Other, and 1.1\% Unknown or unreported. Comparing these percentages to the demographic makeup of the total participant pool at both time points revealed no large differences. We included an investigation of demographic effects in our preliminary analyses. ${ }^{1}$ 


\section{Procedure}

Data collection took place during daytime sessions that included time for breakfast, lunch, and dinner, and breaks for recreation, short movies, college information sessions, and a college tour. Over the course of the research endeavor, participants were provided with a $\$ 50$ gift card to Target, Old Navy, or Best Buy. Students received an additional \$20 in gift cards if they returned rating forms filled out by their parents.

\section{Measures}

Meaning in life-Participants' meaning in life was assessed using three out of the four items from the Persist subscale SAPQ (Chen et al., 2015; "I feel my life has a sense of purpose", "My life feels worthwhile", "I believe there is a larger reason or purpose for my life"; $1=$ not at all, $4=\mathrm{a}$ lot; $a=.78$ ). The fourth item from the Persist subscale, "I feel my life is going nowhere" (reverse-scored), led to marked decreases in internal consistency when included with the other three items for our sample - perhaps due to being reversedscored - and was not used for our meaning in life variable.

Shifting - This variable was made up of the items from the Shift subscale of the SAPQ, a $=.80$ (Chen et al., 2015), and used the same response scale anchors as the Persist subscale items comprising the meaning in life variable. For the prompt "Rate how much you do each of the following when something stressful happens in your life", participants responded to the items "I think about what I can learn from the situation", and "I think about the positive aspects, or the good that can come from the situation." For the prompt "Rate how much you do each of these things when things don't go the way you want and you are not able to change it", participants responded to "I think about what good things could come from the situation" and "I think about what I can learn from the situation" $(a=.91)$.

Depression symptomatology-The Child Depression Inventory (CDI; Kovacs, 1992) was used to assess participants' depressive symptoms. The CDI is a 27 -item self-report measure designed for use with school-aged children and adolescents. Each item represents a depressive symptom with three levels of symptom severity, and respondents choose the level of severity that best describes how they have been feeling over the past two weeks. The CDI includes five subscales: Negative Mood, Interpersonal Problems, Ineffectiveness, Anhedonia, and Negative Self-Esteem. Sample items include "I am sad all the time", "I am tired all the time", and "Nobody really loves me." Reliability and validity are wellestablished for the CDI (Kovacs, 1992, Mattison, Handford, Kales, Goodman, \& McLaughlin, 1990; Smucker, Craighead, Craighead, \& Green, 1986; first wave $a=.88$; second wave $a=.91$ ).

Stress exposure-The Urban Adolescent Life Experiences Scale (UALES; Allison et al., 1999) design is based on the Adolescent Perceived Events Scale (Compas, Davis, Forsythe

\footnotetext{
${ }^{1}$ The total participant pool recruited at the first time point was 50.6\% female, 27.6\% Black or African-American, $15.4 \%$ Hispanic or Latino/a, 14.0\% White or Caucasian, 9.3\% Asian or Asian-American, 0.4\% American Indian or Alaskan Native, 28.2\% Bi-Racial or Multi-Racial, 0.9\% Other, and 3.9\% Unknown or unreported. The total participant pool recruited at the second time point was 57.2\% female, 31.3\% Black or African-American, 16.9\% Hispanic or Latino/a, 12.4\% Asian or Asian-American, 9.5\% White or Caucasian, 27.4\% Bi-Racial or Multi-Racial, 2.0\% Other, and 0.5\% Unknown or unreported.
} 
\& Wagner, 1987). The UALES is an 87-item measure, and each item was generated using data from low-income urban, predominantly ethnic minority, youth (Allison et al., 1999), appropriate for our sample's heterogeneous background makeup. The original measure includes positive and negative events. In the present study, the measure was shortened to include only negative events, as positive events have not been shown to predict psychological problems (Siegel \& Brown, 1988). Respondents rate their frequency of exposure to a series of stressful experiences $(1=$ never, $5=$ every day; major life item "A friend has died"; daily hassle item "I have transportation problems"; first wave $a=.83$; second wave $a=.81$ ).

High consistency among the 87 assessed stressors may arguably point to a general lack of resources or abundance of obstacles that a person faces. Nonetheless, specifically targeting the presence and frequency of major and minor stressor exposure has been shown to be appropriate and informative for examining how stress in a person's life impacts their wellbeing (DeLongis, Coyne, Dakof, Folkman, \& Lazarus, 1982).

\section{Plan of Analysis}

After first assessing our data for completeness, normality, and demographic effects, we then carried out our main analyses. We identified 177 participants who provided data at both waves of data collection. Given that depression may develop over a period of time and have many contributing stressors, we first examined the stress-depression relationship at both time points of data collection by calculating bivariate correlations between first wave stress and first wave depression, and between second wave stress and second wave depression. We also were interested in how stress exposure and depression symptomatology progressed as individual variables from the first time point to the second. To assess this, we calculated bivariate correlations between first wave depression and second wave depression, and between first wave stress exposure and second wave stress exposure. By examining these longitudinal autocorrelations, we aimed to better understand how chronic these conditions are for our participants, and assess the longitudinal stability of our stress exposure and depression symptomatology variables.

To investigate the buffering role of meaning in life and shifting in the stress-depression link, we examined the relationships between participants' meaning in life, shifting, stress exposure and depression in the second wave data using correlational analyses. To further explore these relationships, and thus to theoretically examine the possibility that adolescents may use meaning in life for stability in two different ways, two moderated regression analyses were planned. Levels of depression at the first data collection were included in these models as well to control for the longitudinal aspect of variations in depression. Thus, one moderated regression included stress exposure, meaning in life, the interaction of stress exposure and meaning in life, and first wave depression as predictors for second wave depression. A second moderated regression included stress exposure, shifting, the interaction of stress exposure and shifting, and first wave depression as predictors for second wave depression. Follow-up simple slopes analyses were planned to probe any significant interaction effects that emerged. 


\section{Results}

\section{Preliminary Analyses}

Means for depression and stress exposure at the first wave of data collection, and meaning in life, shifting, depression, and stress exposure at the second wave of data collection were created using a listwise inclusion criterion. We next compared the distribution of our four variables against a normal distribution. Our sample size exceeds the range in which significance testing of skewness and kurtosis is informative, and visual consultation of P-P plots is instead advised in such cases (Field, 2009). These plots revealed all four interest variables to be sufficiently close to the expected values under a normal distribution.

In order to assess the presence of possible gender, race, and age effects in our intended analyses, we ran four ANOVAs, one for each of our second time point variables of interest, with these demographic variables as fixed factors. No gender or race effects were found. However, there was a main effect of age on stress exposure, $F(8,163)=3.21, p=.01$. Therefore, we included age as a predictor in our moderated regression analyses.

\section{Main Analyses}

For the participants that provided data at both time points, first and second wave depression symptomatology and first and second wave stress exposure variables, means, standard deviations, skewness, and zero-order correlations are reported in Table 1. As predicted from the established stress-depression link in the literature, stress exposure and depression symptomatology were strongly positively correlated at both time points. Both waves' depression symptomatology scores were highly positively correlated with one-another, as were both waves' stress exposure scores. To assess the threat of attrition impact to the generalizability of our findings we also looked at the relationship between the stress exposure and depressive symptomatology variables for the entire first wave sample. The detected positive correlation between these variables at time one remained when considering the entire sample.

For all variables at the second time point, as well as depression at the first time point to inform how it relates to second time point variables, means, standard deviations, skewness, and zero-order correlations are reported in Table 2. Consistent with our expectations, higher meaning in life and higher shifting were associated with lower depression.

A moderated regression analysis was used to test for an interaction between stress exposure and meaning in life while controlling for depression at the first time point to account for the longitudinal nature of the study. Age effects were also controlled for by including age at the second time point in the model. Due to missing data for at least one variable, for this analysis $N$ was 161 . For the overall model, $R^{2}=.61$. Depression at time one significantly positively predicted depression at time two, $B=.55, S E=.07, t=7.90, p<.001$. Stress exposure at time two significantly predicted depression at time two, $B=.43, S E=.07, t=$ $5.56, p<.001$, paralleling the positive association between stress and depression in the literature. Higher meaning in life significantly predicted lower depression at time two, $B=$ $-.05, S E=.02, t=-2.60, p=.01$, echoing findings in the literature of a negative relationship between meaning in life and depression. No significant main effect for age was found on 
depression at time two, $B=-.01, S E=.01, t=-.98, p=.33$. Importantly, the interaction between stress exposure and meaning in life was a significant predictor of depression at time two, $B=-.30, S E=.09, t=-3.36, p=.001$. Complete results for this analysis are reported in Table 3.

A simple slopes analysis investigated the detected interaction between stress exposure and meaning in life on depression by assessing how stress exposure predicted depression when meaning in life was at one standard deviation below and above its mean. Figure 1 displays the results of this analysis, and additionally displays the main effect of stress exposure when meaning in life was at its mean. Exposure to stress was associated with depression at low levels of meaning in life, $B=.68, S E=.10, t=6.88, p<.001$. This influence of stress on depression disappeared when values of meaning in life were high, $B=.18, S E=.11, t=$ $1.60, p=.11$. Thus, it seems that at high levels meaning in life may neutralize the otherwise strong relationship between stress and depression outcomes.

A moderated regression analysis was used to also test for an interaction between stress exposure and shifting while controlling for depression at time one and age at time two. Due to missing data for at least one variable, for this analysis $N$ was 157 . For the overall model, $R^{2}=.58$. Depression at time one significantly positively predicted depression at time two, $B$ $=.60, S E=.07, t=8.37, p<.001$. Stress exposure at time two again significantly predicted depression at time two, $B=.46, S E=.07, t=5.94, p<.001$. Higher shifting significantly predicted lower depression at time two, $B=-.04, S E=.02, t=-2.62, p=.02$, suggesting that shifting maybe a useful tool against depression. No significant main effect for age was found on depression at time two, $B=-.01, S E=.01, t=-0.89, p=.38$. The interaction between stress exposure and shifting was not a significant predictor of depression at time two, $B=-.12, S E=.09, t=-1.45, p=.16$. Complete results for this analysis are reported in Table 4.

\section{Discussion}

In the current study we examined the role of meaning in life in the relationship between stress and depression in an adolescent sample. While conceptually replicating the stressdepression link established in the literature, we found evidence for a moderation of that link by meaning in life. At two time points stress exposure was highly predictive of depressive symptomatology in this adolescent sample, while - when measured at the second time point - meaning in life markedly moderated the effect of stress exposure on depression. Importantly, in a prospective design where we were able to control for depression as a prior symptom at time one, meaning accounted for unique variance in depression at time two, suggesting that meaning can impact change in depression outcomes.

The novel contributions of the current study are twofold. The first lies in elucidating the role of meaning in life in the link between stress and depression, with the longitudinal measurement of stress exposure and depression further strengthening this argument. That is, the relationship between exposure to stressful life events and depression levels was detected over two time-points and meaning in life showed a moderating attenuation of this enduring relationship. This supports the role of meaning in life as a resource for resilience against 
stressors. The second major contribution is our demonstration of these patterns in adolescents. As a malleable cognitive-emotional framework, meaning in life may be particularly effective in addressing the threat of stress to adolescents. Youths may benefit from meaning in life by using it to gain cognitive control over stressors they cannot directly influence. Accordingly, participants who reported higher meaning in life and more reframing of stressful and uncontrollable events in the context of their broader meaning in life (shifting) had better depression outcomes. Having a strong sense of meaning in life and being able to flexibly rely on it for stability seems to be protective for this at-risk population.

\section{Theoretical Implications}

Meaning as a buffer for stress-Our finding of meaning in life buffering stressor impact is congruent with theory on the stress-depression link, in which stressors that threaten self-worth promote depressive thinking (Beck, 1983; Blatt, 1974; Hammen, 2005). Under the generic cognitive model, stressful events are appraised with regard to a person's goals, identity, and understanding of their life (Beck, 1983; Beck \& Haigh, 2014). If a stressor conflicts with or disrupts one of these central aspects it may be particularly destabilizing or distressing, a disorientation that is often the case with trauma (e.g. Park, Mills, \& Edmondson, 2012). Cognitive processing of trauma makes the trauma consistent with established meaning in life and cognitive schemas (Lepore, Ragan, \& Jones, 2000; Taylor, 1983). If a person can cope with stress through meaning-making, framing it in a way that integrates into their understanding of self and the world, the stressor may pose less of a threat (Greenberg, 1995; Hembree \& Foa, 2000). Understanding adversity through personal narrative theoretically aids in establishing indirect control (Heckhausen \& Schulz, 1995). Thus, meaning in life may be well suited for coping by providing avenues for psychological control over situations that cannot be directly changed, supported by our findings of better depression outcomes associated with the tendency to engage in shifting. Furthermore, associations between meaning in life and decreased psychopathology suggest the importance for having meaning in life in general. In other words, a secure meaning narrative may serve both as a source of general stability and for reframing specific stressors in tumultuous circumstances. The finding of a moderating role of meaning in life in the stress-depression relationship, and no moderation of this relationship by shifting, suggests that in this sample high meaning in life might have been more important than shifting as a coping method in resisting stress.

Unique stressors and resources in adolescence-Finding meaning in life to buffer stressor impact on adolescents' depression is of high importance due to the vulnerability of this age group. Described widely as a time of storm and stress, adolescence is characterized by identity exploration and social role change. Youths are also at heightened risk of stress exposure and depression (Thapar et al., 2012), with neurological change rendering them especially sensitive to the impact of stress and psychosocial factors (Andersen \& Teicher, 2008). With the challenges of role, identity, and responsibility negotiation compounded upon possible general stressors in an adolescent's environment (Eccles et al., 2003; Steinberg \& Morris, 2001), this period of life deserves particular attention. As such, adaptive methods of coping with stress in adolescence have potential for lifelong impact and informing coping later in life. 
Further, well-being and meaning in life may interact in a unique way during adolescence. Having achieved commitment to life purpose has been found to fully mediate the relationship between identity commitment and a variety of well-being indices in two samples of adolescents and one sample of emerging adults (Burrow \& Hill, 2011). Additionally, recent studies suggest that adolescents experience benefits from searching for meaning in life, with increases to positive health behaviors and fewer problem behaviors (Brassai et al., 2012). In adults, searching for meaning in life has been shown to predict decreased subjective well-being unless the individual already is bolstered by possessing high levels of meaning in life (Cohen \& Cairns, 2012; Park et al., 2010). This indicates that adolescents may have an orientation to meaning in life that addresses the identity needs of their developmental stage and is particularly flexible in its ability to provide support. Our findings of meaning in life as a moderator of stress exposure in adolescents indicate that at this age individuals already have access to using their understanding of meaning in life for resilience.

\section{Practical Implications}

The weakened impact of stress exposure on depressive symptoms in participants with high meaning in life is compelling support for meaning in life as an effective coping resource for adolescents. Meaning-making coping may thus be similarly effective in adolescents as in adults (Harris et al., 2011; Park et al., 2008; Park, 2005). This is potentially relevant to interventions in adolescents at risk for increased psychopathology and a history of stress or trauma. Evidence-supported interventions for increasing life meaning have involved reminiscing on and reviewing important periods of participants' lives (Westerhof, Bohlmeijer, \& Valenkamp, 2004; Westerhof, Bohlmeijer, van Beljouw, \& Pot, 2010) and exploring important relationships and orientation to crises (Cho et al., 2013). Given that many adolescents may not have a wealth of important life periods on which to reminisce, and that some may have a limited number of impactful relationships, these interventions would need to be tailored appropriately. Encouragingly, recent research has found adolescent meaning in life to be bolstered when youths identify strongly with their present identity commitments and when less rumination is engaged during identity exploration (NegruSubtirica, Pop, Luyckx, Dezutter, \& Steger, 2016). This suggests that adolescents can access meaning in life in a way that is characteristic of their developmental period. In addition, according to evidence linking adolescent well-being with engaging in a search for meaning in life (Brassai et al., 2012), youths may respond positively to interventions designed to help them initiate a search for meaning in life or accomplish a combination of searching for increased meaning in life while connecting with understandings of life meaning they may already have. Further exploration attempting to identify especially powerful sources of life meaning for adolescents (i.e. relationships, roles, identity symbols) would be particularly useful for designing meaning-focused interventions specific to this age group.

\section{Limitations}

Although participants endorsed the full range of depression symptomatology severity, the sample mean centered in low-to-moderate severity values. Generalizing our findings to severely depressed individuals may be limited, though meaning in life has been associated with decreased depression symptoms in clinically depressed samples previously (Thakur \& 
Basu, 2010). In addition, while convergent validity for the SAPQ in capturing adolescent meaning in life has been supported (Chen et al., 2015), our findings would be further strengthened by future replications using additional instruments to capture adolescent meaning in life.

The cross-sectional nature of the meaning in life and shifting variables is a limitation in drawing causal conclusions about our relationships of interest. However, our finding of moderation of the stress-depression relationship through meaning in life should be interpreted in light of the existing literature that has demonstrated the enduring nature of the stress-depression link through longitudinal designs (Hammen, 1991). Also, in the current study participants reported whether they had ever been exposed to a given stressor, suggesting that - at least to some extent - the data shed light on life meaning's influence in the stress-depression link beyond assessments of the participants' stress levels at the time of the study. Of need for further investigation is the remaining question of whether meaning in life directly attenuates the stress-depression relationship or whether people who possess meaning in life are otherwise especially resistant to stressor impact.

As another caveat to interpreting our findings in accordance with our hypothesized directional relationships, it is important to keep in mind that stress and depression can be mutually-reinforcing. Research on the stress generation hypothesis suggests that there is a more complex relationship between stress and depression than the one we have used to construct our models (Hammen, 2006; Hammen, 1991). Specifically, depressed individuals seem to play a participatory role in their environments in such a way that engenders stressful interactions and occurrences. Given that we used depression as an outcome of stress in our theoretical and analytical models, it is possible that some of the depression variance is selfreinforcing by increasing depressed individuals' likelihood to encounter stress. However, controlling for depression at the first time point allows us to be more confident that the depression symptom variance explained by stress exposure can be attributed to stressor impact rather than stress that has been created by prior depressive patterns.

\section{Conclusions}

The current research suggests that meaning in life is important for the affective well-being of adolescents who have encountered and continue to encounter stressful experiences.

Remarkably, it seems that high levels of meaning in life may be able to efficiently buffer the impact of stress exposure in terms of depression severity. This adds support to the growing literature on the adolescent and adult health benefits of having a firm sense that one's life is meaningful. Additionally, these patterns represent encouraging evidence that adolescents, despite being in the midst of social and identity upheaval, have access to strategies for supporting their own feelings of psychological control and agency.

\section{Acknowledgments}

This project was supported by funding from NIH Grant R21 AA021073 awarded to Dr. Emma Adam and Dr. Kathryn Grant. 


\section{References}

Achenbach, T. Manual for the Youth Self-Report and 1991 Profile. Burlington, VT: University of Vermont Department of Psychiatry; 1991.

Allison K, Burton L, Marshall S, Perez-Febles A, Yarrington J, Kirsh L, Merriwether DeVries C. Life experiences among urban adolescents: Examining the role of context. Child Development. 1999; 70:1017-1029. [PubMed: 10446733]

Andersen SL, Teicher MH. Stress, sensitive periods and maturational events in adolescent depression. Trends in Neurosciences. 2008; 31:183-191. [PubMed: 18329735]

American Psychiatric Association. Diagnostic and statistical manual of mental disorders. 5. Washington, DC: Author; 2013.

Beck, AT. Cognitive therapy of depression: New perspectives. In: Clayton, PJ., Barrett, JE., editors. Treatment of depression: Old controversies and new approaches. New York: Raven Press; 1983.

Beck AT, Haigh EA. Advances in cognitive theory and therapy: The generic cognitive model. Annual Review of Clinical Psychology. 2014; 10:1-24.

Beck R, Perkins TS. Cognitive content-specificity for anxiety and depression: A meta-analysis. Cognitive Therapy and Research. 2001; 25:651-663.

Blatt SJ. Levels of object representation in anaclitic and introjective depression. The Psychoanalytical Study of the Child. 1974; 29:7-157.

Bohlmeijer E, Smit F, Cuijpers P. Effects of reminiscence and life review on late-life depression: a meta-analysis. International Journal of Geriatric Psychiatry. 2003; 18:1088-1094. [PubMed: 14677140]

Brassai L, Piko B, Steger M. Existential attitudes and Eastern European adolescents' problem and health behaviors: Highlighting the role of the search for meaning in life. The Psychological Record. 2012; 62:719-734.

Braet C, Wante L, Van Beveren ML, Theuwis L. Is the cognitive triad a clear marker of depressive symptoms in youngsters? European Child \& Adolescent Psychiatry. 2015; 24:1261-1268. [PubMed: 25583656]

Burrow AL, Hill PL. Purpose as a form of identity capital for positive youth adjustment. Developmental Psychology. 2011; 47:1196-1206. [PubMed: 21574703]

Chen E, McLean K, Miller G. Shift-and-persist strategies: Associations with socioeconomic status and the regulation of inflammation among adolescents and their parents. Psychosomatic Medicine. 2015; 77:371-382. [PubMed: 26167560]

Cho S, Bernstein K, Roh S, Chen D. Logo-autobiography and its effectiveness on depressed Korean immigrant women. Journal of Transcultural Nursing. 2013; 24:33-42. [PubMed: 22802301]

Cohen K, Cairns D. Is searching for meaning in life associated with reduced subjective well-being? Confirmation and possible moderators. Journal of Happiness $\backslash$ Studies. 2012; 13:313-331.

Compas B, Davis G, Forsythe C, Wagner B. Assessment of major and daily stressful events during adolescence: The Adolescent Perceived Events Scale. Journal of Consulting and Clinical Psychology. 1987; 55:534-541. [PubMed: 3624609]

Currier J, Holland J, Chisty K, Allen D. Meaning made following deployment in Iraq or Afghanistan: Examining unique associations with posttraumatic stress and clinical outcomes. Journal of Traumatic Stress. 2011; 24:691-698. [PubMed: 22113939]

DeLongis A, Coyne JC, Dakof G, Folkman S, Lazarus RS. Relationship of daily hassles, uplifts, and major life events to health status. Health Psychology. 1982; 1:119-136.

Disner SG, Beevers CG, Haigh EA, Beck AT. Neural mechanisms of the cognitive model of depression. Nature Reviews Neuroscience. 2011; 12:467-477. [PubMed: 21731066]

Durlak J. Relationship between individual attitudes toward life and death. Journal of Consulting and Clinical Psychology. 1972; 38:463. [PubMed: 5032556]

Eccles, J., Templeton, J., Barber, B., Stone, M. Adolescence and emerging adulthood: The critical passage ways to adulthood. In: Bornstein, MH.Davidson, LC.Keyes, LM., Moore, KA., editors. Well-being: Positive development across the lifespan. Mahwah, NJ: Erlbaum; 2003.

Field, A. Discovering statistics using SPSS. Sage publications; 2009. 
Greenberg MA. Cognitive processing of traumas: The role of intrusive thoughts and reappraisals. Journal of Applied Social Psychology. 1995; 25:1262-1296.

Halama P, Dedová M. Meaning in life and hope as predictors of positive mental health: Do they explain residual variance not predicted by personality traits? Studia Psychologica. 2007; 49:191200.

Hammen C. Generation of stress in the course of unipolar depression. Journal of Abnormal Psychology. 1991; 100:555-561. [PubMed: 1757669]

Hammen C. Stress and depression. Annual Review of Clinical Psychology. 2005; 1:293-319.

Hammen C. Stress generation in depression: Reflections on origins, research, and future directions. Journal of Clinical Psychology. 2006; 62:1065-1082. [PubMed: 16810666]

Harris JI, Erbes CR, Engdahl BE, Thuras P, Murray-Swank N, Grace D, Le T. The effectiveness of a trauma focused spiritually integrated intervention for veterans exposed to trauma. Journal of Clinical Psychology. 2011; 67:425-438. [PubMed: 21294116]

Heckhausen J, Schulz R. A life-span theory of control. Psychological Review. 1995; 102:284-304. [PubMed: 7740091]

Hembree EA, Foa EB. Posttraumatic stress disorder: Psychological factors and psychosocial interventions. The Journal of Clinical Psychiatry. 2000; 61:1-478.

Ho M, Cheung F, Cheung S. The role of meaning in life and optimism in promoting well-being. Personality and Individual Differences. 2010; 48:658-663.

Hong L. College stress and psychological well-being: Self-transcendence meaning of life as a moderator. College Student Journal. 2008; 42:531-541.

Hsieh H, Wang J. Effect of reminiscence therapy on depression in older adults: a systematic review. International Journal of Nursing Studies. 2003; 40:335-345. [PubMed: 12667510]

Kiang L, Fuligni AJ. Meaning in life as a mediator of ethnic identity and adjustment among adolescents from Latin, Asian, and European American backgrounds. Journal of Youth and Adolescence. 2010; 39:1253-1264. [PubMed: 19915965]

Kleftaras G, Psarra E. Meaning in life, psychological well-being and depressive symptomatology: A comparative study. Psychology. 2012; 3:337-345.

Kovacs, M. Children's Depression Inventory. New York, NY: Multi-Health Systems; 1992.

Krause N. Stressors arising in highly valued roles, meaning in life, and the physical health status of older adults. The Journals of Gerontology: Social Sciences. 2004; 59:287-297.

Larner B, Blow A. A model of meaning-making coping and growth in combat veterans. Review of General Psychology. 2011; 15:187.

Lepore SJ, Ragan JD, Jones S. Talking facilitates cognitive-emotional processes of adaptation to an acute stressor. Journal of personality and social psychology. 2000; 78:499-508. [PubMed: 10743876]

Lewinsohn PM, Rohde P, Seeley JR. Major depressive disorder in older adolescents: Prevalence, risk factors, and clinical implications. Clinical Psychology Review. 1998; 18:765-794. [PubMed: 9827321]

Lyon D, Younger J. Purpose in life and depressive symptoms in persons living with HIV disease. Journal of Nursing Scholarship. 2001; 33:129-133. [PubMed: 11419307]

Martela F, Steger MF. The three meanings of meaning in life: Distinguishing coherence, purpose, and significance. The Journal of Positive Psychology. 2016; 11:531-545.

Mascaro N, Rosen D. Existential meaning's role in the enhancement of hope and the prevention of depressive symptoms. Journal of Personality. 2005; 73:985-1014. [PubMed: 15958142]

Mascaro N, Rosen D. Assessment of existential meaning and its longitudinal relations with depression symptoms. Journal of Social and Clinical Psychology. 2008; 27:576-599.

Mattison R, Handford H, Kales H, Goodman A, McLaughlin R. Four-year predictive value of the Children's Depression Inventory. A Journal of Consulting and Clinical Psychology. 1990; 2:169_ 174.

Mazure CM. Life stressors as risk factors in depression. Clinical Psychology: Science and Practice. 1998; 5:291-313. 
Monroe, SM., Hadjiyannakis, K. The social environment and depression: Focusing on severe life stress. In: Gotlib, IH., Hammen, CL., editors. Handbook of Depression. New York: Guilford Press; 2002.

Negru-Subtirica O, Pop EI, Luyckx K, Dezutter J, Steger MF. The meaningful identity: A longitudinal look at the interplay between identity and meaning in life in adolescence. Developmental Psychology. 2016; 52:1926-1936. [PubMed: 27598255]

Owens G, Steger M, Whitesell A, Herrera C. Posttraumatic stress disorder, guilt, depression, and meaning in life among military veterans. Journal of Traumatic Stress. 2009; 22:654-657. [PubMed: 19924820]

Park C. Religion as a meaning-making framework in coping with life stress. Journal of Social Issues. 2005; 61:707-729.

Park C, Edmondson D, Fenster J, Blank T. Meaning making and psychological adjustment following cancer: The mediating roles of growth, life meaning, and restored just-world beliefs. Journal of Consulting and Clinical Psychology. 2008; 76:863-875. [PubMed: 18837603]

Park CL, Mills MA, Edmondson D. PTSD as meaning violation: Testing a cognitive worldview perspective. Psychological Trauma: Theory, Research, Practice, and Policy. 2012; 4:66-73.

Park N, Park M, Peterson C. When is the search for meaning related to life satisfaction? Applied Psychology: Health and Well-Being. 2010; 2:1-13.

Paykel ES. Life events and affective disorders. Acta Psychiatrica Scandinavica. 2003; 108:61-66. [PubMed: 12807378]

Reker G. The purpose-in-life test in an inmate population: An empirical investigation. Journal of Clinical Psychology. 1977; 33:688-693. [PubMed: 893692]

Reker G, Cousins J. Factor structure, construct validity and reliability of the Seeking of Noetic Goals (SONG) and purpose in Life (PIL) Tests. Journal of Clinical Psychology. 1979; 35:85-91.

Robbins M, Francis LJ. Religion, personality, and well-being: The relationship between church attendance and purpose in life. Journal of Research on Christian Education. 2000; 9:223-238.

Siegel J, Brown J. A prospective study of stressful circumstances, illness symptoms, and depressed mood among adolescents. Developmental Psychology. 1988; 24:715-721.

Simonelli L, Fowler J, Maxwell GL, Andersen B. Physical sequelae and depressive symptoms in gynecologic cancer survivors: Meaning in life as a mediator. Annals of Behavioral Medicine. 2008; 35:275-284. [PubMed: 18386113]

Smucker M, Craighead E, Craighead L, Green B. Normative and reliability data for the Children's Depression Inventory. Journal of Abnormal Child Psychology. 1986; 14:25-39. [PubMed: 3950219]

Southwick S, Gilmartin R, McDonough P, Morrissey P. Logotherapy as an adjunctive treatment for chronic combat-related PTSD: A meaning-based intervention. American Journal of Psychotherapy. 2006; 60:161-174. [PubMed: 16892952]

Steger, M. Meaning in life. In: Lopez, SJ., editor. Oxford Handbook of Positive Psychology. 2. Oxford, UK: Oxford University Press; 2009.

Steger MF, Frazier P, Oishi S, Kaler M. The meaning in life questionnaire: Assessing the presence of and search for meaning in life. Journal of Counseling Psychology. 2006; 53:80-93.

Steinberg L, Morris AS. Adolescent development. Journal of Cognitive Education and Psychology. $2001 ; 2: 55-87$.

Sullivan PF, Neale MC, Kendler KS. Genetic epidemiology of major depression: Review and metaanalysis. American Journal of Psychiatry. 2000; 157:1552-1562. [PubMed: 11007705]

Tavernier R, Willoughby T. Adolescent turning points: The association between meaning-making and psychological well-being. Developmental Psychology. 2012; 48:1058-1068. [PubMed: 22122472]

Taylor SE. Adjustment to threatening events: A theory of cognitive adaptation. American Psychologist. 1983; 38:1161-1173.

Tedeschi RG, Calhoun LG. Posttraumatic growth: Conceptual foundations and empirical evidence. Psychological Inquiry. 2004; 15:1-18.

Thakur K, Basu S. A probe of existential meaning in depression. Somatic Inkblot Society Journal of Projective Psychology and Mental Health. 2010; 907:56-62. 
Thapar A, Collishaw S, Pine DS, Thapar AK. Depression in adolescence. The Lancet. 2012; 379:1056-1067.

Updegraff JA, Silver RC, Holman EA. Searching for and finding meaning in collective trauma: Results from a national longitudinal study of the 9/11 terrorist attacks. Journal of Personality and Social Psychology. 2008; 95:709-722. [PubMed: 18729704]

Werner FM, Coveñas R. Classical neurotransmitters and neuropeptides involved in major depression in a multi-neurotransmitter system: a focus on antidepressant drugs. Current Medicinal Chemistry. 2013; 20:4853-4858. [PubMed: 24083608]

Westerhof G, Bohlmeijer E, Valenkamp M. In search of meaning: A reminiscence program for older persons. Educational Gerontology. 2004; 30:751-766.

Westerhof G, Bohlmeijer E, van Beljouw I, Pot AM. Improvement in personal meaning mediates the effects of a life review intervention on depressive symptoms in a randomized controlled trail. The Gerontologist. 2010; 50:541-549. [PubMed: 20045873]

Yalom, I. Existential Psychotherapy. New York: Basic Books; 1980.

Zika S, Chamberlain K. Relation of hassles and personality to subjective well-being. Journal of Personality and Social Psychology. 1987; 53:135-162.

Zika S, Chamberlain K. On the relation between meaning in life and psychological well-being. British Journal of Psychology. 1992; 83:133-145. [PubMed: 1559144] 


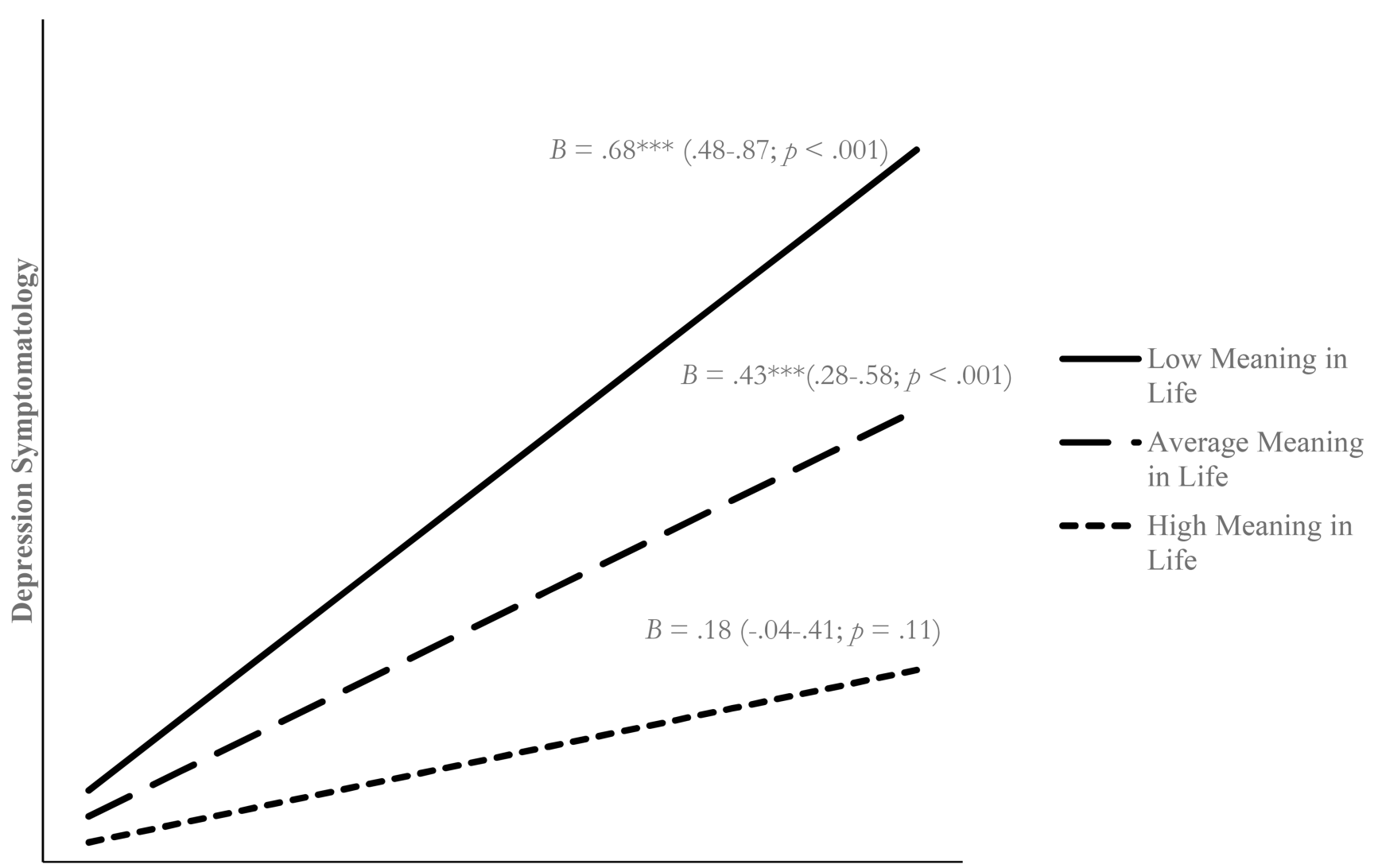

Stress Exposure

Figure 1.

The interaction of meaning in life $\mathrm{x}$ stress exposure influences mean depression levels at the second time point of data collection, when controlling for depression at time one and age at time two.

Note. $* * * p<.001$; parentheses following betas represent confidence intervals and exact $p$ values; degrees of freedom for each simple effects test are $(5,155)$. 


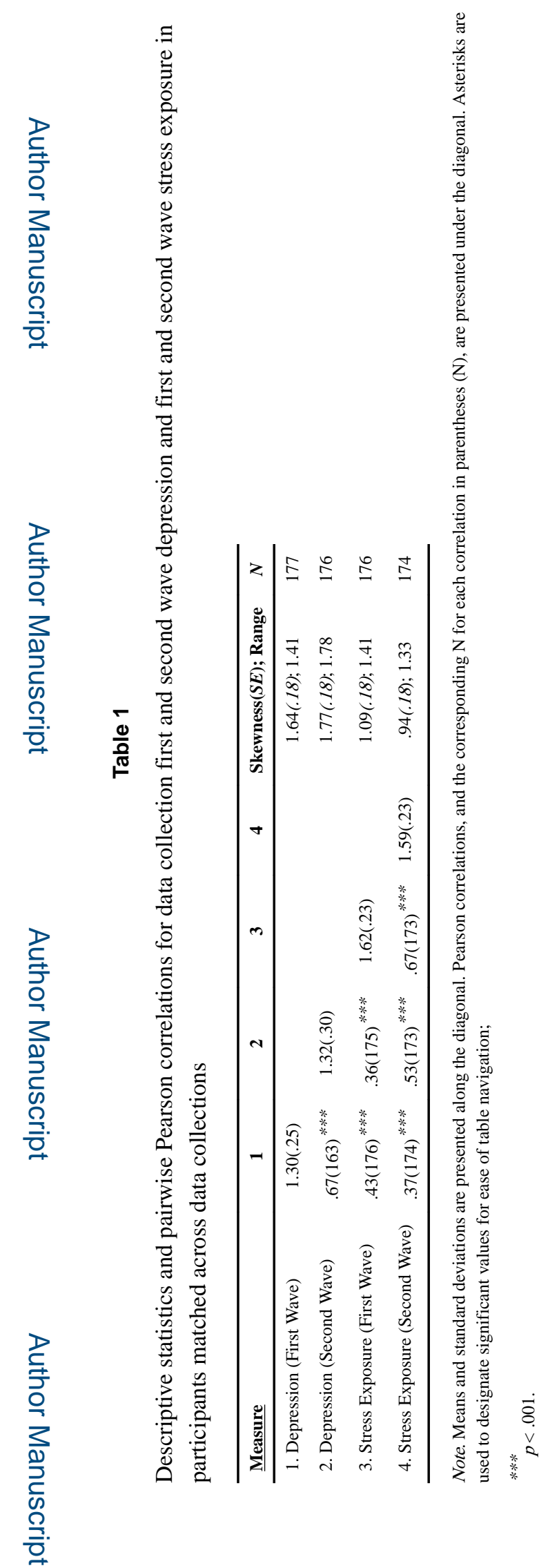




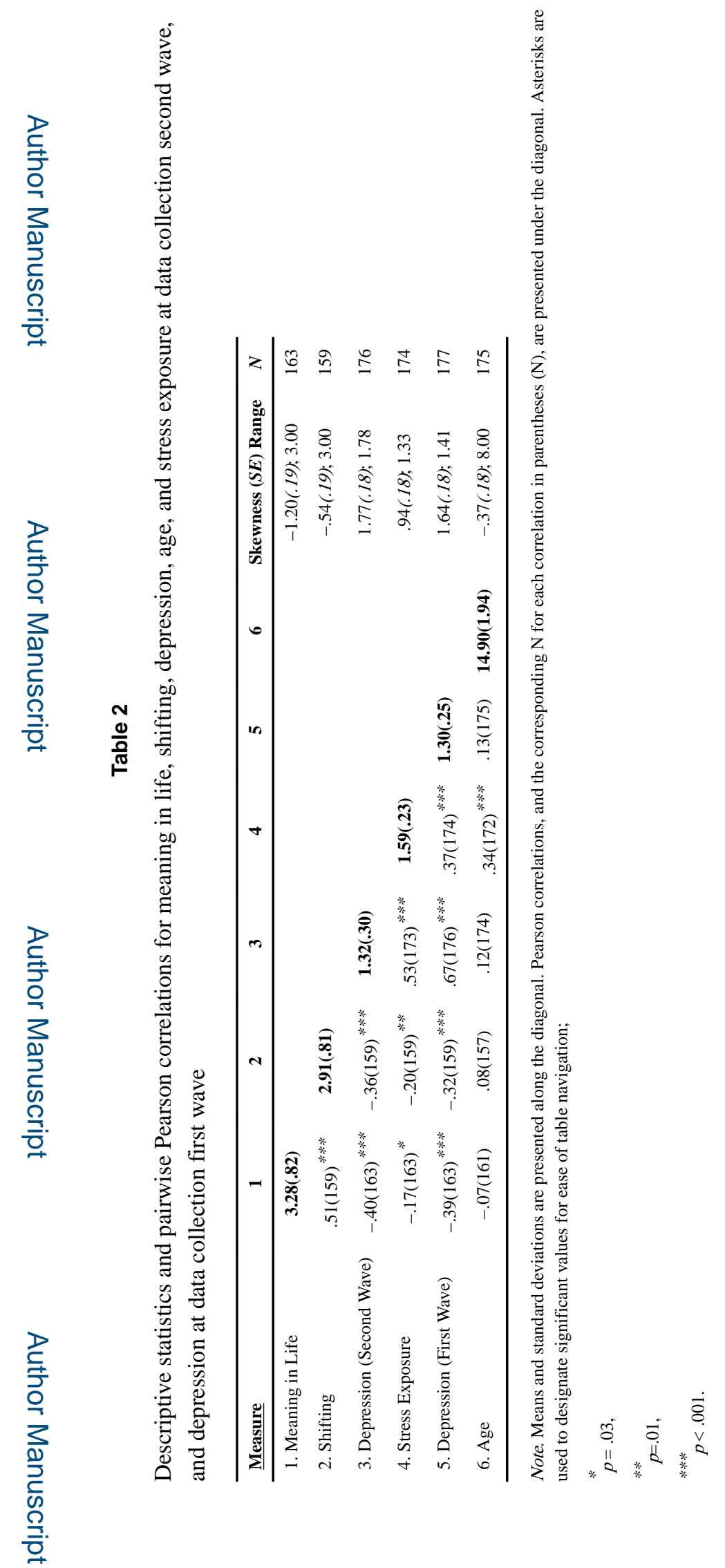

J Adolesc. Author manuscript; available in PMC 2019 June 01. 


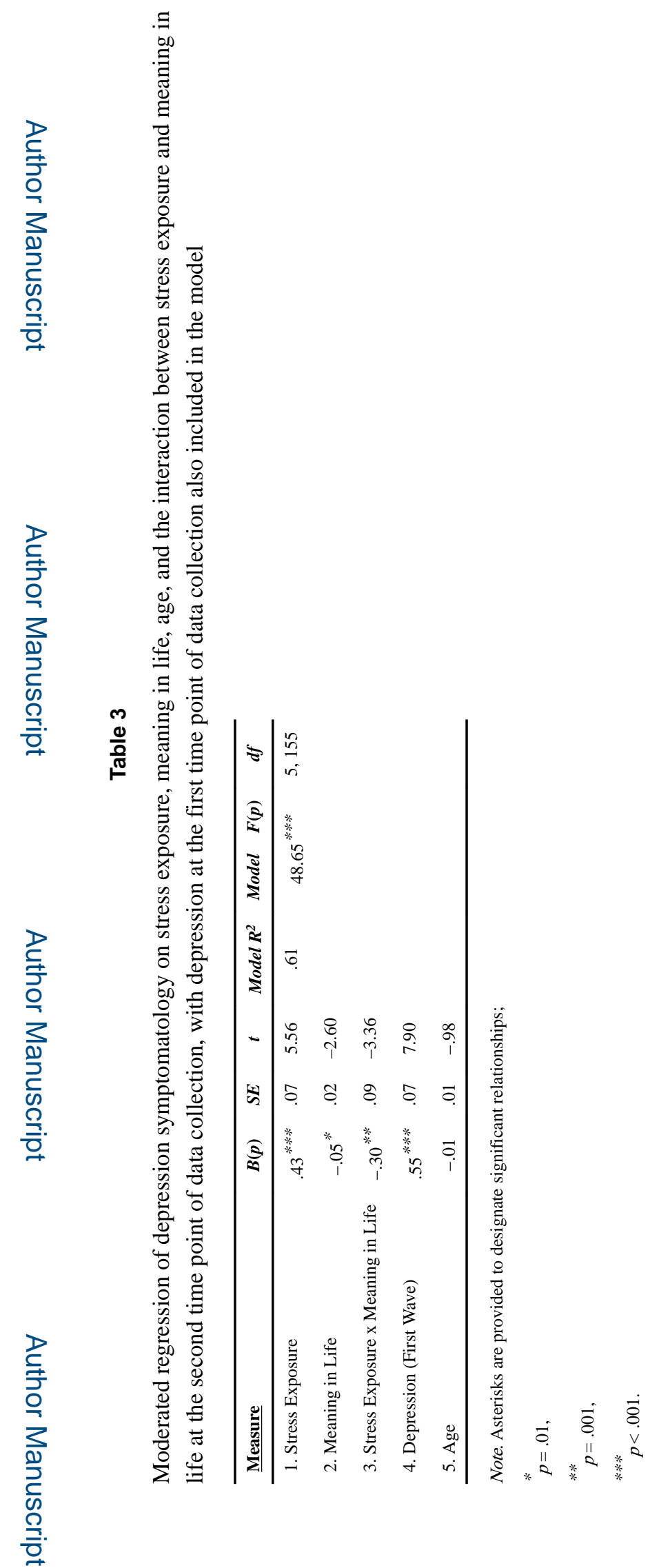

J Adolesc. Author manuscript; available in PMC 2019 June 01. 


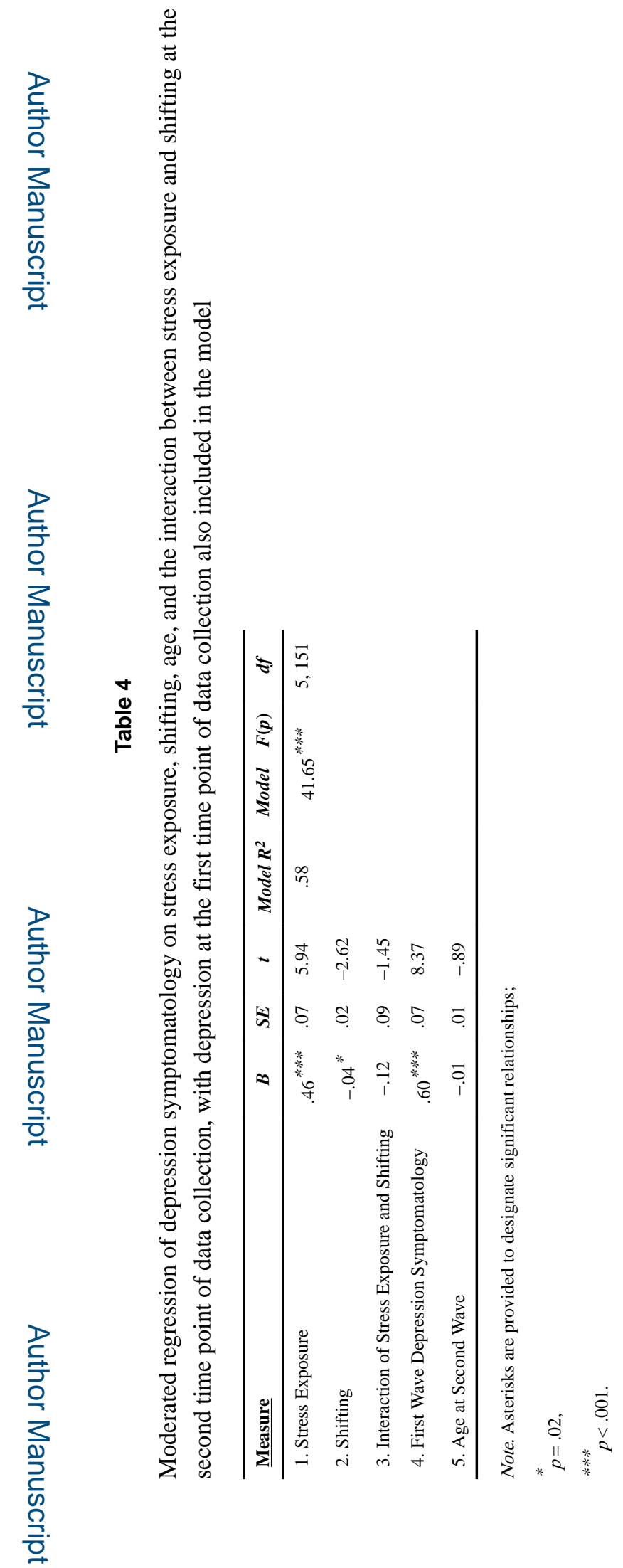

J Adolesc. Author manuscript; available in PMC 2019 June 01. 